\title{
The BRICS in the international money and debt capital markets
}

\author{
Viacheslav M. Shavshukov \\ St. Petersburg State University, St. Petersburg, Russia
}

\section{Keywords}

BRICS, new phenomenon in finance and the global economy, international money, debt and capital markets

\begin{abstract}
This paper discusses the role of the BRICS countries as strong players in the global economy and is aimed at identifying major obstacles to their economic growth. It examines money and debt capital markets in the BRICS and their attractiveness to foreign investment in 2006-2016. The research methodology is based on a comparative analysis of financial market indicators.

During the period 2006-2007 the BRICS countries were becoming increasingly attractive for foreign direct investment, mostly due to their natural and labor resources. Modernization reforms, carried out in the 80s, also stimulated competition and enhanced standards of living. The BRICS countries achieved higher GDPs, promoted production growth and achieved a positive balance of payments. Since 2007 the BRICS have not only attracted investment, but have been investing in the developed economies.

However, the analysis also revealed some fundamental economic weaknesses, originating in the global economic crisis. The most serious of these are increasing financial risks and the destabilization of the money markets. The post-crises period is characterized by the struggle for stability and the creation of new infrastructure (New Development Bank and Pool of Contingent Reserve Arrangement).
\end{abstract}

Corresponding author: Viacheslav M. Shavshukov

Email addresses for corresponding author: shavshukov@rambler.ru

First submission received: $12^{\text {th }}$ September 2017

Revised submission received: $14^{\text {th }}$ October 2017

Accepted: $2^{\text {nd }}$ November 2017

\section{Acknowledgement}

This paper was supported by Natalia Zalutskaya, St. Petersburg State University

\section{Introduction}

\section{The BRICS as a new player in the global economy}

The BRICS as a recognized global entity represent one of the driving forces in the world economy. It is a union of the most dynamically developing economies which includes Brazil, Russia, India, China and South Africa. The term "BRIC" was first introduced into political life by Jim O'Neil, the leading analyst of Goldman Sachs investment bank, in 2001. Among international financial market traders, the term was used mainly to identify the assets of those countries. The first methodological study of the BRICs was published only in 2005 (Jim O'Niel, Dominic Wison, 2005). Goldman Sachs carried out a methodological analysis and identified three groups of factors which affect the economic growth in the BRICs: position in the world economy and trade; world market capitalization (as a share (\%) of the world GDP and trade) and FDI inflows; 13 indicators of the Growth Environmental Score. On 18 th February 2011 the Republic of South Africa accessed the BRIC and the new union received the name "BRICS".

The BRICS is not an economic union like the EC, but rather a political club and alliance with the aim of becoming a new power in geo-economics and geopolitics. There is some justification for such an ambition, since these countries occupy $26 \%$ of the Earth's surface, have $43 \%$ of the population, produce about $15 \%$ of the world's GDP, $40 \%$ of the wheat, $50 \%$ of the pork, and $30 \%$ of the beef and poultry, from $32 \%$ of the world's agricultural land. Russia, China and India are armed with 5,190 nuclear weapons. Also, these countries benefit from: hydrocarbon reserves in Russia, relatively cheap labour in China, manufacturing in India (IT production), South African natural resources, and Brazilian domination in the production of sugar, soybean, iron ore, bio fuel, ethanol and electric power. 
In the 1980's two parallel processes took place in the world economy: globalization and modernization of the four most dynamically developing world economies of Brazil, Russia, India and China. These four countries started to reform in the 1980s; the first phase of globalization started at the beginning of the same decade. It was the period when Russia and China rejected the command economy and Brazil and India maintained their market economy and the four countries began their reforms towards the more efficient "mixed economy". Reforms in the BRIC countries differed in their content, form, phases, and national goals, but the vector of the reforms was directed at creating competitive national economies and businesses, enhancement of living standards, and establishing a new axis in the world's geo-economics and geopolitics.

At the beginning of the $21^{\text {st }}$ century global competition took a new turn. However, the BRIC countries had maintained their advantages in terms of GDP growth (8.4\% in 2007), industrial production (5-13\%), trade, international financing and, finally, a positive balance of payments (ongoing budget surpluses).

Leading BRIC companies, such as Embraer (Brazil), Gasprom, Severstal, Rosneft, RZD ("Russian Railways"), VTB, Suhoy (Russia), Essar Steel, Steel Author, Tata (India), were becoming more and more competitive and some of them developed into transnational corporations (TNCs) and multinational banks (TNBs). Many were entering international money and capital markets and taking part in syndicated capital borrowings and placements (IPOs) with record breaking sums of \$8-9 bln.

Emerging economies became increasingly attractive for direct investment from the financial centre, for example, in the Shanghai Free Economic Zone alone there were about 3,000 international companies. India was the fourth among High-Tech and R\&D counties with Oracle, Intel, Adobe, St Microelectronics and SAP, while such companies as Texas Instruments, HP, Microsoft, GE, Phillips, Motorola, Google, Cisco, Bayer, and Siemens were increasingly employing talented IT specialists from China, India and Russia.

However, this rapid growth was impeded by fundamental economic weaknesses, such as low quality and effectiveness of corporate and state management, especially when compared to the developed countries. For example, in 2009 Russia was placed $60^{\text {th }}$ in the sphere of innovation, China - 81 $1^{\text {st }}$, India $109^{\text {th }}$ (KAM, 2011). Reasons included: strengthening national currencies, increasing inflation (up to $6 \%$ in investment goods, up to $12 \%$ in consumer ones), salary growth $(9 \%)$, increasing productivity, low competitiveness and weak banking systems.

\section{The BRICS in the global financial markets \\ 1.1. The BRICS in the international money market}

One of the first signs of established global finance is the lowering or elimination of barriers between national and international financial markets. It is achieved through liberalization of exchange regulations, creation of proper market infrastructure and elimination of excessive administrative obstacles. Significant lowering of barriers results in lower money market rates, followed by lower pegged prices. Thus, money market rates decreased by over two thirds, down from $24.62 \%$ in 1996 to $7.53 \%$ in 2006, government bond rates - by almost two thirds from 13.66\% in 2002 to $4.84 \%$ in 2011 (International Financial Statistics, 2002; International Financial Statistics, 2006.) The trend of lowering lending rates continued: from 1996 to 2009 the average rate in the BRICS decreased by 3.2 times from $51.64 \%$ to $16.16 \%$ (to compare: the average lending rate in the USA during the same period was 10.6\%) (The World Bank Data, 2011; International Financial Statistics, 2002).

During the decade preceding the global economic crisis, emerging countries, especially the BRICS, acted as a driving force for the growth of the world economy. It was their need for investment that stimulated the increase in the value of dollar credit to emerging market economies from $\$ 92 \mathrm{bln}$. in 1996 to $\$ 300$ bln. in 2010 and \$3.3 trln in 2016 (International Banking and financial market developments, 1997.- p. 10; BIS 80th Annual Report, 2010. - p 47; BIS Quarterly Review, September 2016. - A5). Since the amount of TNC borrowing is significantly higher than the syndication of emerging market companies, their share in the period went down from $16 \%$ to $11 \%$. First the BRICS and then other emerging countries demonstrated great resilience during the post-crisis period; they overcame the crisis faster than developed economies, resulting in a $19 \%$ increase of their share of a total volume of syndicated borrowings on the international 
money market, with the BRICS share of $41 \%$ (\$110.2 bln.) and India and Russia being the major borrowers with \$33bln. (BIS Quarterly Report 2006: A82; BIS Quarterly Review 2011: A110).

\subsection{The BRICS on Debt Capital Markets}

As a source of long term investment the debt and capital markets remain the largest and most attractive, as is shown in Table 1.

Table 1. International market of debt securities in 2005 and 2010, \$ bln.

\begin{tabular}{|c|c|c|c|c|c|c|c|c|}
\hline \multirow[t]{3}{*}{ Countries } & \multicolumn{2}{|c|}{$\begin{array}{l}\text { Total volume of capital issue, } \\
\text { \$ bln. }\end{array}$} & \multicolumn{6}{|c|}{$\begin{array}{l}\text { Volume and structure of the market, } \\
\text { share by states and institutions, } \%\end{array}$} \\
\hline & \multirow[b]{2}{*}{2005} & \multirow[b]{2}{*}{2010} & \multicolumn{2}{|c|}{ State } & \multicolumn{2}{|c|}{ Financial institutions } & \multicolumn{2}{|c|}{ Corporations } \\
\hline & & & 2005 & 2010 & 2005 & 2010 & 2005 & 2010 \\
\hline \multirow[t]{2}{*}{ All countries } & 14634.5 & 29045.6 & 1437.0 & 2598.7 & 11105.9 & 21709.0 & 1547.2 & 3760.3 \\
\hline & $100 \%$ & $100 \%$ & $9.8 \%$ & $9.3 \%$ & $75.9 \%$ & $74.7 \%$ & $10.6 \%$ & $13 \%$ \\
\hline \multirow{2}{*}{$\begin{array}{l}\text { Developed } \\
\text { countries }\end{array}$} & 13044.5 & 26169.5 & 953.7 & 1971.0 & 10707.67 & 20810.2 & 1383.3 & 3388.3 \\
\hline & $89.1 \%$ & $90.0 \%$ & $6.5 \%$ & $6.8 \%$ & $3.2 \%$ & $71.6 \%$ & $9.5 \%$ & $11.7 \%$ \\
\hline \multirow{2}{*}{$\begin{array}{l}\text { Developing } \\
\text { countries }\end{array}$} & 862.8 & 1635.3 & 452.5 & 587.7 & 273.8 & 721.2 & 136.6 & 326.5 \\
\hline & $5.9 \%$ & $5.6 \%$ & $3.0 \%$ & $2.0 \%$ & $1.9 \%$ & $2.5 \%$ & $0.9 \%$ & $1.1 \%$ \\
\hline \multirow{2}{*}{$\begin{array}{l}\text { Offshoring } \\
\text { centres }\end{array}$} & 182.7 & 263.2 & 30.8 & 40.0 & 124.5 & 177.6 & 27.3 & 45.5 \\
\hline & $1.25 \%$ & $0.9 \%$ & $0.2 \%$ & $0.1 \%$ & $0.8 \%$ & $0.6 \%$ & $0.2 \%$ & $0.2 \%$ \\
\hline $\begin{array}{c}\text { BRICS } \\
\text { including: }\end{array}$ & & $541.3(1.9 \%)$ & & - & & $336.3(1.2 \%)$ & & $\begin{array}{l}100.2 \\
0.4 \%\end{array}$ \\
\hline Brazil & & $186.7(0.6 \%)$ & & $51.0(0.2 \%)$ & & $108.7(0.4 \%)$ & & $27.0(0.1 \%)$ \\
\hline Russia & & $162.7(0.56 \%)$ & & $31.6(0.1 \%)$ & & $105.8(0.36 \%)$ & & $25.4(0.08 \%)$ \\
\hline India & & $52.4(0.2 \%)$ & & $-5.9(0.02 \%)$ & & $23.9(0.1 \%)$ & & $28.5(0.1 \%)$ \\
\hline China & & $87.0(0.4 \%)$ & & $11.1(0.03 \%)$ & & $69.7(0.2 \%)$ & & $11.5(0.03 \%)$ \\
\hline RSA & & $52.5(0.2 \%)$ & & - & & $28.2(0.1 \%)$ & & $7.8(0.026 \%)$ \\
\hline
\end{tabular}

Source: (BIS Quarterly Review, 2016, pp. A86-A90; A113-A117).

The state remains the main issuer in the emerging countries. As the main economic agent in developing economies, it evokes more trust with TNC and conservative funds. It is interesting to note that in 2005 the issued volume of BRIC government bonds was only half that of the developed countries (\$453 bln. against $\$ 954$ bln.).

The total volume of investment into developing countries doubled during the 6 years (2000-2006) from $\$ 216$ to $\$ 411$ bln. It was clear evidence of growing trust, as a result of an increase in credit ratings, profitability and liquidity. The BRICS had become increasingly attractive to TNCs, Mutual Funds and Hedge funds. The international debt capital markets opened the door to business units from BRICS economies because their capitalization, rating history, transparency, and stability were become larger, bigger, better, and more. The initial entry to the international markets was connected with the issue of Eurobonds (See Table 2). In 2006-2007 the average volume of issue was $\$ 500 \mathrm{mln}$., period of maturity 5-7 years, coupon 150-200 b.p. over U.S. T-Bills, that adequately reflected the sovereign and corporate ratings.

Table 2. Eurobond issues of BRICS corporations and financial institutions, 2006, 2014/2015*

\begin{tabular}{|c|c|c|c|c|c|c|c|c|c|}
\hline \multirow[b]{2}{*}{ Country } & \multicolumn{3}{|c|}{2006} & \multicolumn{6}{|c|}{$2014 / 2015$} \\
\hline & Issuer/Rating & $\begin{array}{c}\text { Amount } \\
\text { USD, bln. } \\
\text { Date of } \\
\text { maturity }\end{array}$ & Coupon & Issuer / rating & $\begin{array}{c}\text { Amount, } \\
\text { bln. } \\
\text { Currency } \\
\text { Date of } \\
\text { maturity }\end{array}$ & Coupon & $\begin{array}{l}\text { Issue } \\
\text { price }\end{array}$ & Listing & Comments \\
\hline \multirow{4}{*}{ RUSSIA } & Rosselkhozbank & 0.5 & 6.97 & $\begin{array}{c}\text { RZD, Corp } \\
\text { BB+ }\end{array}$ & $\begin{array}{l}5 \text { CHF } \\
(2018)\end{array}$ & 2.177 & 99.888 & $\begin{array}{c}\text { Frankfurt, } \\
\text { Berlin }\end{array}$ & $\begin{array}{c}\text { SPV } \\
\text { RZD Capital } \\
\text { PLC, } \\
\text { Ireland }\end{array}$ \\
\hline & $\begin{array}{l}\text { Alpha Bank } \\
\text { (BB-) }\end{array}$ & 0.4 (2009) & 7.875 & $\begin{array}{c}\text { RZD, Corp } \\
\text { BB+ }\end{array}$ & $\begin{array}{l}5 \text { CHF } \\
(2021)\end{array}$ & 3.374 & 100 & $\begin{array}{c}\text { Dublin, } \\
\text { Frankfurt, } \\
\text { Berlin, } \\
\text { Munich }\end{array}$ & $\begin{array}{c}\text { SPV } \\
\text { RZD Capital } \\
\text { PLC, } \\
\text { Ireland }\end{array}$ \\
\hline & Mosenergo (B) & $\begin{array}{c}0.5 \\
(2011)\end{array}$ & 7.68 & Gazprom BB+ & $\begin{array}{l}\text { NA USD } \\
(2034)\end{array}$ & 8.625 & 100 & Luxemburg & $\begin{array}{c}\text { SPV } \\
\text { Gaz capital } \\
\text { SA } \\
\text { Euroclear, } \\
\text { Clearstream }\end{array}$ \\
\hline & Alliance Oil (B+) & 0.3 (2011) & 9.40 & $\begin{array}{c}\text { Sibur } \\
\text { Securioties } \\
\text { Ltd } \\
\text { BB+ }\end{array}$ & NA USD & 3.914 & 100 & Luxemburg & $\begin{array}{c}\text { Cyprus Co } \\
\text { SPV Ireland } \\
\text { Euroclear, } \\
\text { Clearstream }\end{array}$ \\
\hline
\end{tabular}

www.jbrmr.com A Journal of the Academy of Business and Retail Management (ABRM) 


\begin{tabular}{|c|c|c|c|c|c|c|c|c|c|}
\hline & $\begin{array}{l}\text { Russian } \\
\text { Standard Bank } \\
(\mathrm{B}+)\end{array}$ & 0.650 (2009) & 6.825 & $\begin{array}{c}\text { Vnesheconom } \\
\text { bank } \\
\text { BB+ }\end{array}$ & $\begin{array}{l}\text { NA USD } \\
(2023)\end{array}$ & 5.942 & 100 & Luxemburg & $\begin{array}{c}\text { SPV VEB } \\
\text { Finance PLC } \\
\text { Ireland } \\
\text { Euroclear, } \\
\text { Clearstream }\end{array}$ \\
\hline \multirow{4}{*}{ BRAZIL } & Braskem $(\mathrm{BB}+)$ & $0.275(2008)$ & 8 & $\begin{array}{c}\text { Petrobras } \\
\text { Global } \\
\text { Finance BV }\end{array}$ & $\begin{array}{c}0.2 \text { USD } \\
(2024)\end{array}$ & 6.25 & 99.772 & NA & $\begin{array}{c}\text { SPV } \\
\text { Netherland }\end{array}$ \\
\hline & Banko Votorantim & $0.2(2016)$ & $\begin{array}{c}150 \text { b.p. } \\
\text { over US } \\
\text { T-Bills }\end{array}$ & $\begin{array}{c}\text { Vale Overseas } \\
\text { LTD } \\
\text { BBB }\end{array}$ & $\begin{array}{l}0.660 \\
\text { USD } \\
(2032)\end{array}$ & 4.375 & 98.804 & NA & $\begin{array}{l}\text { SPV Cayman } \\
\text { Islands } \\
\text { Euroclear, } \\
\text { Clearstream }\end{array}$ \\
\hline & BicBanco (Ba3) & $0.2(2009)$ & 8.50 & $\begin{array}{c}\text { Centrals } \\
\text { Eletricas } \\
\text { Brasileiras SA } \\
\text { BB+ } \\
\end{array}$ & $\begin{array}{c}0.3 \text { USD } \\
(2021)\end{array}$ & 5.75 & 100 & NA & $\begin{array}{c}\text { Euroclear, } \\
\text { Clearstream }\end{array}$ \\
\hline & $\begin{array}{l}\text { Banko Cruzeiro } \\
\text { do Sul }\end{array}$ & $0.1(2011)$ & 8.125 & $\begin{array}{c}\text { Banco do } \\
\text { Brazil SA } \\
\text { BB- } \\
\end{array}$ & 0.2 USD & 9 & 100 & NA & $\begin{array}{c}\text { SPV Cayman } \\
\text { Euroclear, } \\
\text { Clearstream }\end{array}$ \\
\hline \multirow[t]{3}{*}{ CHINA } & $\begin{array}{l}\text { Golden Eagle } \\
\text { Retail Group }\end{array}$ & 1 (2011) & NA & $\begin{array}{c}\text { Eastern } \\
\text { Creation II } \\
\text { Investment } \\
\text { holdings Ltd } \\
\text { A } \\
\end{array}$ & $\begin{array}{l}\text { NA EUR } \\
(2018)\end{array}$ & 1 & NA & $\begin{array}{l}\text { Dublin Hong } \\
\text { Kong }\end{array}$ & British Virgin \\
\hline & Chinhan Bank & $0.350(2015)$ & $\begin{array}{c}205 \text { b.p. } \\
\text { over US } \\
\text { T-bills }\end{array}$ & Suedost bahn & $\begin{array}{l}\text { NA CHF } \\
(2032)\end{array}$ & 1.25 & 99.421 & NA & $\begin{array}{l}\text { SPV } \\
\text { Swiss }\end{array}$ \\
\hline & & & & $\begin{array}{c}\text { Transocean } \\
\text { Inc } \\
\text { BB+ } \\
\end{array}$ & $\begin{array}{l}\text { NA USD } \\
(2017)\end{array}$ & 3 & 99.714 & NA & $\begin{array}{l}\text { SPV Cayman } \\
\text { Islands }\end{array}$ \\
\hline \multirow[t]{2}{*}{ INDIA } & $\begin{array}{l}\text { Bank of India } \\
(\mathrm{BB}+)\end{array}$ & $\begin{array}{l}240 \mathrm{mln} . \\
(2021)\end{array}$ & 6 & $\begin{array}{c}\text { Bank of India } \\
\text { BBB- }\end{array}$ & $\begin{array}{l}\text { NA USD } \\
(2019)\end{array}$ & 3.125 & 99.574 & NA & $\begin{array}{c}\text { SPV Jersey } \\
\text { Euroclear, } \\
\text { Clearstream }\end{array}$ \\
\hline & & & & $\begin{array}{c}\text { Bharat } \\
\text { Petroleum } \\
\text { Corp } \\
\text { BBB- } \\
\end{array}$ & $\begin{array}{l}\text { NA USD } \\
(2025)\end{array}$ & 4 & 99.105 & NA & $\begin{array}{c}\text { Euroclear, } \\
\text { Clearstream }\end{array}$ \\
\hline \multirow[t]{2}{*}{ RSA } & & & & $\begin{array}{c}\text { Transnet SOC } \\
\text { Ltd } \\
\text { BBB } \\
\end{array}$ & NA USD & 4.5 & 99.472 & $\begin{array}{c}\text { LSE } \\
\text { FSE } \\
\text { Stuttgart SE }\end{array}$ & \\
\hline & & & & $\begin{array}{c}\text { Edcon } \\
\text { Holding Ltd } \\
\text { CCC- }\end{array}$ & $\begin{array}{l}\text { NA EUR } \\
(2019)\end{array}$ & 13.375 & 100 & NA & $\begin{array}{c}\text { Euroclear, } \\
\text { Clearstream }\end{array}$ \\
\hline
\end{tabular}

*Sources: (International Financing Review. - Sept., 23, 2006. - P.88; International Financing Review. Sept., 9, 2006. - P.91-92; Bloomberg professional, April 29, 2015).

During and after the Global Crises Eurobonds were not the main sources of funding for the BRICS economies. The share of issues from the developed economies was $99 \%$ and $99 \%$ were USD denominated. But we see the first issues in CHY and ZAR, and maturity dates were increased from 5 to 10 years. The prices of issue were comparable 98.8 (BRICS) vs 97.9-100 (developed economies). All issues used the EuroClear and Clear Stream clearing systems, but the majority of BRICS' issues were structured either as offshore companies from the Cayman Islands, the British Virgin Islands, Cyprus or SPVs in the Netherlands or Ireland. The coupons of BRICS' issues were double that of companies from developed economies (4-13\% vs $0.75-4 \%)$. Stock markets of the emerging economies during that period were adequately developed and had relatively modern infrastructures with high profitability and liquidity, which made them attractive for global investors. Assets of such leading transnational banks as $H S B C$, Citigroup, and UBS were located in some 80 countries around the world. By 2000 the economies of developed and developing countries had attracted average annual investment flows amounting to \$200700 bln.

Table 3 demonstrates the progress of BRIC companies in the debt securities and capital markets by 2006: their IPOs were placed in the London and Hong Kong financial centres and on the financial periphery in Brazil, accompanied by a GDR option, and leading investment banks such as UBS, CS, Merrill Lynch together with an Indian banker Kotak Mahitra acted as book runners for IPOs. 
Table 3. IPO/Secondary, Public offering/private placement of BRICS Co on the Emerging and Developed Capital Markets, 2006, 2014*

\begin{tabular}{|c|c|c|c|c|c|c|c|c|}
\hline \multirow[t]{2}{*}{ Country } & Issuer & $\begin{array}{l}\text { Amn., } \\
\text { bln. } \\
\text { USD }\end{array}$ & Type of deal & $\begin{array}{l}\text { Book } \\
\text { runner }\end{array}$ & Issuer & $\begin{array}{l}\text { Amn., } \\
\text { Cur }\end{array}$ & $\begin{array}{c}\text { Type of } \\
\text { Deal }\end{array}$ & Book runner \\
\hline & \multicolumn{4}{|c|}{2006} & \multicolumn{4}{|c|}{2014} \\
\hline \multirow[t]{3}{*}{ INDIA } & $\begin{array}{l}\text { Power } \\
\text { finance } \\
\text { Corp }\end{array}$ & 0.321 & $\begin{array}{l}\text { IPO } \\
\text { Privatization } \\
\text { according } \\
\text { Government } \\
\text { plan }\end{array}$ & $\begin{array}{l}\text { Enam } \\
\text { Financial } \\
\text { Consultants, } \\
\text { Kotak } \\
\text { Mahintra }\end{array}$ & $\begin{array}{l}\text { Adlabs } \\
\text { Entertainment }\end{array}$ & $\begin{array}{l}4.3 \mathrm{bln} \\
\text { INR }\end{array}$ & $\begin{array}{l}\text { IPO } \\
\text { Natl India } \\
20.3 \text { mln. } \\
\text { ordinary } \\
\text { shares }\end{array}$ & $\begin{array}{l}\text { Kotak Mahindra } \\
\text { Capital Co } \\
\text { SPV Deutscher } \\
\text { Equities India Pte } \\
\text { Ltd }\end{array}$ \\
\hline & $\begin{array}{l}\text { Cairn } \\
\text { Energy } \\
\text { India }\end{array}$ & 2 & IPO LSE & $\begin{array}{l}\text { ABN AMRO, } \\
\text { Merrill Lynch }\end{array}$ & $\begin{array}{l}\text { HDFC Bank } \\
\text { Ltd }\end{array}$ & $\begin{array}{l}1.3 \mathrm{bln} . \\
\text { USD }\end{array}$ & $I P O$ & $\begin{array}{l}\text { Goldman Sachs, } \\
\text { Barclays, } \\
\text { Mahindra Bank Ltd }\end{array}$ \\
\hline & $\begin{array}{l}\text { Kalpataru } \\
\text { PowerTran } \\
\text { smission }\end{array}$ & 0.075 & $\begin{array}{l}\text { Private } \\
\text { Qualified } \\
\text { Institutions } \\
\text { Placement } \\
\text { with an } \\
\text { option } \\
\text { ADR/GDR } \\
\text { markets }\end{array}$ & $\begin{array}{l}\text { Kotak } \\
\text { Mahindra }\end{array}$ & $\begin{array}{l}\text { Bharti } \\
\text { Infratel Ltd }\end{array}$ & $\begin{array}{l}311 m l n . \\
I N R\end{array}$ & $\begin{array}{l}\text { Secondary } \\
144 A\end{array}$ & $\begin{array}{l}\text { UBS, } \\
\text { Bank of America }\end{array}$ \\
\hline \multirow[t]{3}{*}{ BRAZIL } & $\begin{array}{l}\text { Medial } \\
\text { Saude }\end{array}$ & 0.300 & $\begin{array}{l}\text { IPO } \\
\text { (First } \\
\text { healthcare } \\
\text { deal } \\
\text { secondary) }\end{array}$ & Credit Suisse & $\begin{array}{l}\text { Ouro Fino } \\
\text { Saude } \\
\text { Animal } \mathrm{Pa}\end{array}$ & $\begin{array}{l}363.460 \\
\text { mln. } \\
\text { BRL }\end{array}$ & $\begin{array}{l}\text { IPO } \\
\text { Bovespa, } \\
13,46 \mathrm{mln} \\
\text { Ord. } \\
\text { shares }\end{array}$ & Banko do Brazil SA \\
\hline & $\begin{array}{l}\text { Electropaul } \\
o \\
\text { Metropolit } \\
\text { ana }\end{array}$ & 0.533 & $\begin{array}{l}\text { IPO } \\
\text { (secondary) } \\
\text { Level } 2 \\
\text { listing on } \\
\text { the Bovespa }\end{array}$ & $\begin{array}{l}\text { Credit Suisse, } \\
\text { JPMorgan }\end{array}$ & $\begin{array}{l}\text { Garnero } \\
\text { Group } \\
\text { Acquisition }\end{array}$ & $\begin{array}{l}125 \text { mln. } \\
\text { USD }\end{array}$ & $\begin{array}{l}\text { IPO } \\
\text { NASDAQ } \\
12.5 \mathrm{mln} \\
\text { Ord. } \\
\text { shares }\end{array}$ & $\begin{array}{l}\text { Early Bird Capital } \\
\text { Inc }\end{array}$ \\
\hline & & & & & $\begin{array}{l}\text { Porto Sudeste } \\
\text { VM SA }\end{array}$ & $\begin{array}{l}188 \mathrm{mln} \\
\text { BRL }\end{array}$ & Secondary & Credit Suisse \\
\hline \multirow[t]{3}{*}{ CHINA } & $\begin{array}{l}\text { Action } \\
\text { Semicondu } \\
\text { ctor }\end{array}$ & 0.083 & $\begin{array}{l}\text { IPO } \\
\text { (primary) }\end{array}$ & $\begin{array}{l}\text { Citigroup, } \\
\text { Morgan } \\
\text { Stanley }\end{array}$ & $\begin{array}{l}\text { Alibaba } \\
\text { Group } \\
\text { Holding } \\
\text { Internet Co }\end{array}$ & $\begin{array}{l}21.7672 \\
\text { bln. } \\
\text { USD }\end{array}$ & $\begin{array}{l}\text { IPO } \\
\text { NYSE }\end{array}$ & $\begin{array}{l}\text { Lead manager } \\
\text { Credit Suisse } \\
\text { Co-lead manager } \\
\text { books: } \\
\text { Deutsche Bank } \\
\text { Goldman Sachs } \\
\text { JP Morgan Securitie } \\
\text { Morgan Stanley } \\
\text { Citi } \\
\text { BOCI Asia Ltd } \\
\text { China International } \\
\text { Capital Corp HK } \\
\text { Security } \\
\text { CLSA Ltd } \\
\text { DBS Bank } \\
\text { HSBC Bank PLC } \\
\text { Mizuho Bank Ltd } \\
\text { Pacific Crest } \\
\text { Securities }\end{array}$ \\
\hline & $\begin{array}{l}\text { Beijing } \\
\text { North Star }\end{array}$ & 0.461 & $\begin{array}{l}\text { IPO } \\
\text { (primary) }\end{array}$ & Galaxy & $\begin{array}{l}\text { Jumei } \\
\text { International } \\
\text { Holding } \\
\text { Retail }\end{array}$ & $\begin{array}{l}204 \mathrm{mln} . \\
\text { USD }\end{array}$ & IPO NYSE & Goldman Sachs \\
\hline & $\begin{array}{l}\text { China Blue } \\
\text { Chemical }\end{array}$ & 0.341 & $\begin{array}{l}\text { IPO } \\
\text { (primary) }\end{array}$ & JPMorgan & $\begin{array}{l}\text { Fuyao Glass } \\
\text { Industry } \\
\text { Group Co }\end{array}$ & $\begin{array}{l}951.9 \\
m l n . \\
H K D\end{array}$ & $\begin{array}{l}I P O \\
144 A\end{array}$ & $\begin{array}{l}\text { HSBC } \\
\text { JP Morgan } \\
\text { UBS }\end{array}$ \\
\hline
\end{tabular}




\begin{tabular}{|c|c|c|c|c|c|c|c|c|}
\hline & $\begin{array}{l}\text { Focus } \\
\text { Media }\end{array}$ & 0.140 & $\begin{array}{l}\text { IPO } \\
\text { (secondary) }\end{array}$ & Credit Suisse & $\begin{array}{l}\text { Industrial } \\
\text { Bank Co Ltd }\end{array}$ & $\begin{array}{l}3.7 \text { bln. } \\
\text { CHY }\end{array}$ & $\begin{array}{l}\text { IPO, } \\
\text { Private } \\
\text { placement }\end{array}$ & Goldman Sachs \\
\hline & $\begin{array}{l}\text { Hopson } \\
\text { Developme } \\
n t\end{array}$ & 0.104 & $\begin{array}{l}\text { IPO } \\
\text { (primary) }\end{array}$ & Credit Suisse & $\begin{array}{l}\text { Wintime } \\
\text { Energy Co } \\
\text { Ltd }\end{array}$ & $\begin{array}{l}1.6 \mathrm{bln} . \\
\mathrm{CHY}\end{array}$ & $\begin{array}{l}\text { IPO China } \\
\text { A Shares }\end{array}$ & $\begin{array}{l}\text { Bank of America, } \\
\text { UBS, Morgan } \\
\text { Stanley, JP Morgan, } \\
\text { Nomura, Credit } \\
\text { Swiss } \\
\end{array}$ \\
\hline & $\begin{array}{l}\text { REF } \\
\text { Properties }\end{array}$ & 0.206 & $\begin{array}{l}\text { IPO } \\
\text { (primary) }\end{array}$ & $\begin{array}{l}\text { Morgan } \\
\text { Stanley, } \\
\text { JPMorgan }\end{array}$ & $\begin{array}{l}\text { Goundong } \\
\text { Guanghua } \\
\text { Sci-Tech Co }\end{array}$ & $\begin{array}{l}60.2 \mathrm{mln} . \\
\mathrm{CHY}\end{array}$ & $\begin{array}{l}\text { IPO, China } \\
\text { A Shares }\end{array}$ & GF Securities \\
\hline & $\begin{array}{l}\text { Beijing } \\
\text { Jingkelong }\end{array}$ & 0.076 & $\begin{array}{l}\text { IPO } \\
\text { (primary) } \\
\text { Hong Kong } \\
\text { listing }\end{array}$ & UBS & $\begin{array}{l}\text { Grand Group } \\
\text { Investment } \\
\text { Plc }\end{array}$ & $\begin{array}{l}10.860 \\
m \ln . \\
G B P\end{array}$ & $I P O$ & $N A$ \\
\hline & $\begin{array}{l}\text { China } \\
\text { Merchants } \\
\text { Bank }\end{array}$ & 2.41 & $\begin{array}{l}\text { IPO Hong } \\
\text { Kong listing }\end{array}$ & $\begin{array}{l}\text { UBS, } \\
\text { JPMorgan }\end{array}$ & Wowo Ltd & $\begin{array}{l}40 \mathrm{mln} . \\
\text { USD }\end{array}$ & $I P O$ & $\begin{array}{l}\text { Axiom Capital } \\
\text { Management Inc }\end{array}$ \\
\hline \multirow[t]{2}{*}{ RUSSIA } & $\begin{array}{l}\text { Otkritije } \\
\text { Investicii, } \\
\text { Plc. }\end{array}$ & 0.881 & IPO & $\begin{array}{l}\text { ING, } \\
\text { Renaissance } \\
\text { Capital }\end{array}$ & $\begin{array}{l}\text { Lenta Ltd } \\
\text { Retail }\end{array}$ & $\begin{array}{l}225 \mathrm{mln} . \\
\text { USD }\end{array}$ & $\begin{array}{l}\text { IPO } \\
\text { LSE }\end{array}$ & $\begin{array}{l}\text { Credit Suisse } \\
\text { Securities (Europe) } \\
\text { Ltd } \\
\text { JP Morgan } \\
\text { Securities Plc } \\
\text { VTB Capital Plc } \\
\end{array}$ \\
\hline & $\begin{array}{l}\text { Uralkalij, } \\
\text { Plc. }\end{array}$ & 1 & $\begin{array}{l}\text { IPO } \\
\text { Includes a } \\
\text { GDR listing } \\
\text { at } L S E\end{array}$ & $\begin{array}{l}\text { Credit Suisse, } \\
\text { UBS }\end{array}$ & & & & \\
\hline \multirow[t]{4}{*}{ RSA } & & & & & $\begin{array}{l}\text { Alexander } \\
\text { Forbes Group } \\
\text { Holding } \\
\text { Diversified } \\
\text { Finance Co }\end{array}$ & $\begin{array}{l}3.477 \\
\text { bln. } \\
\text { ZAR }\end{array}$ & $\begin{array}{l}\text { IPO } \\
\text { Johanbln } \\
\text { nesburg SE } \\
431.9 \text { mln. } \\
\text { ordinary } \\
\text { shares }\end{array}$ & $\begin{array}{l}\text { Deutche Securities } \\
\text { Inc }\end{array}$ \\
\hline & & & & & $\begin{array}{l}\text { SA Aspean } \\
\text { Pharmacare } \\
\text { Holdings Ltd }\end{array}$ & $\begin{array}{l}855.7 \\
\text { mln. } \\
\text { ZAR }\end{array}$ & IPO & Citi, UBS \\
\hline & & & & & $\begin{array}{l}\text { Nothan } \\
\text { Platinum Ltd. }\end{array}$ & $\begin{array}{l}202.7 \\
\text { mln. } \\
\text { ZAR }\end{array}$ & Secondary & One capital \\
\hline & & & & & $\begin{array}{l}\text { Novecs } \\
\text { Holdings Ltd. }\end{array}$ & $\begin{array}{l}90.5 \\
\text { mln } \\
\text { ZAR }\end{array}$ & IPO & Investec \\
\hline
\end{tabular}

*Sources: (International Financing Review - 2006, Sept. 9. - P.99-100; Ibid, 2006, Sept. 16. - P.111; Ibid, 2006, Sept.23. - P.104, 106. Bloomberg Professional. April 10, 2015).

Comparative country analyses regarding volume of issues, share of the international market, and number of issuers show that the BRICS outnumbered the issuers from developed markets. Before the Crisis of 2006 Chinese companies carried out 88 issues totalling $\$ 31$ bln with a 7.4\% share of the World market, India - 104 issues, totalling $\$ 9.5$ bln a 1.5\% share, Brazil: 30 issues, $\$ 9.5$ bln and 2.3\% of share and Russia: 14, 15.3, a 3.6\% share. For comparison, US companies in 2006 carried out 436 issues totalling \$94 bln with a market share of $22.4 \%$. The total market share by value of BRICS issues was $14.5 \%$. (IFR, Sept. 2016. - P. 102).

After the Global Crisis (2014) the main sources for funding became the domestic BRICS markets at Johannesburg SE, BOVESPA. Indian companies carried out 42 IPOs and secondary POs at BSE and Natl India. From a list of 178 deals Chinese companies had only one at the NYSE, the others were in - Hong Kong, Shenzhen and Shanghai. It became usual that listing at both the LSE and NYSE, involved underwriting, book running and lead management participation of western investment bankers Credit Suisse, Deutsche Bank, Goldman Sachs, JP Morgan Securities, Morgan Stanley, Citi, HSBC Bank Plc, UBS, and (as new phenomenon) investment bankers from emerging markets: VTB Capital Plc (Russia); BOCI Asia Ltd, Pacific Crest Securities, China International Capital Corp, HK Securiti, Mizuho Bank Ltd, (HK); Relegate capital 
markets, Kotak Mahindra Bank, Sarthi Capital Advisors Ltd, Microsee India Ltd, (India); Dougxing Securities Ltd, China Securities, Huarong Securities Co Ltd, China Lion Securities Ltd, Huafu Securities Ltd (China); Java Capital, One capital (South Africa).

New features of the BRICS stock markets.

Before the Global Crisis we saw a trend for stability in Stock Indexes. In 2006 the range of DJIA, FTSE, and NASDAQ was only $7 \%$, coefficient of correlation $0.9,0.85$ and 0.68 accordingly (see Table 4 ). It was one vector and synchronized motion with the DJIA index dominating as a benchmark. Other turbulent factors such as oil prices and macroeconomic imbalances were not yet apparent.

After the Global Crisis this stability did not persist. In 2014-2015 the situation disimproved. The Index Range became 28\%, Coefficient of correlation 0.1-0.5. We observed negative trends in Indexes in Brazil, Russia and India. What are the general reasons for this situation? It seems these are the shocks on oil and Forex markets, macroeconomic imbalances and significant falls in the traditional benchmarks.

Table 4. Financial indicators of World Top and BRICS Stock Exchanges, 2006-2015*

\begin{tabular}{|c|c|c|c|c|c|c|c|}
\hline \multirow[t]{2}{*}{ Indexes } & \multicolumn{3}{|c|}{2006} & \multicolumn{4}{|c|}{$2012-2015$} \\
\hline & $\begin{array}{c}\text { Changes } \\
\text { between } \\
\text { H/L } \\
\text { rates, } \\
\%, \\
52 \\
\text { Weeks }\end{array}$ & $\begin{array}{c}\text { DIV } \\
\text { (P/E ratio) }\end{array}$ & $\begin{array}{l}\text { Coefficient of } \\
\text { correlation, } \\
\text { compare } \\
\text { with DJIA** }\end{array}$ & $\begin{array}{c}\text { Changes } \\
\text { between } \\
\text { H/L rates, } \\
\%, \\
52 \text { Weeks } \\
2014 / 2015\end{array}$ & $\begin{array}{l}\text { Coefficient of } \\
\text { correlation, } \\
\text { compare with } \\
\text { DJIA, } \\
2014 / 2015^{\star *}\end{array}$ & $\begin{array}{l}\text { Coefficient of } \\
\text { correlation, } \\
\text { compare with } \\
\text { DJIA, } \\
\text { 2006-2015** }\end{array}$ & $\begin{array}{l}\text { Coefficient of } \\
\text { correlation } \\
\text { compare with } \\
\text { DJIA, } \\
\text { 2012-2015** }\end{array}$ \\
\hline DJIA & 11.7 & $\begin{array}{c}0.1-2.9 \\
(18.1)\end{array}$ & 1 & 13.25 & 1 & 1 & 1 \\
\hline FTSE & 12.5 & $\begin{array}{c}0.3-2.2 \\
(25)\end{array}$ & 0.9 & 7.93 & 0.6 & 0.9 & 0.92 \\
\hline NASDAQ & NA & NA & 0.68 & 16.70 & 0.78 & 0.96 & 0.98 \\
\hline Nikkey225 & NA & NA & 0.39 & 38.46 & 0.5 & 0.6 & 0.97 \\
\hline Bovespa & 14 & $3.38(12)$ & 0.8 & 35.6 & 0.7 & 0.06 & -0.6 \\
\hline RTS/MOEX & 16 & $0.82-6.4(16.9)$ & 0.8 & 39.59 & 0.8 & -0.01 & -0.8 \\
\hline BSE Sens. & 16 & $1.15(20.7)$ & 0.9 & 35.24 & -0.2 & 0.8 & 0.9 \\
\hline Shanghai A & 24 & $0.77(23.5)$ & 0.8 & 79.10 & 0.4 & 0.1 & 0.5 \\
\hline $\begin{array}{c}\text { FTSE/JSE } \\
\text { Africa TOP } 40\end{array}$ & 14 & 1.17 (15.34) & 0.9 & 15.62 & 0.7 & 0.9 & 0.9 \\
\hline
\end{tabular}

*Sources: (Bloomberg professional, 2006-2015). Coefficient of correlation was calculated by Pierson.

**Sources: (Financial Times, Jan.20, 2007. - P.10-11; http://www.bloomberg.com/quote/TOP40: IND 09.11.2012;

http://www.jse.co.za/Home/Market_Data.aspx 12.11.2012; Factsheet: FTSE/JSE TOP 40 Index/ p-2;

www.bloomberg.com/quot/indexes/: IND. 17.03.2015; Bloomberg professional, 26.06.2015).

\section{Preliminary conclusions}

Emerging markets had higher returns (2-3 times), but less stability. In 2006 the emerging market's Index by Morgan Stanley Capital International (MSCI) increased by $29 \%$, DJIA BRIC Index - by $53 \%$ (Financial Times. - 2007, Jan 20). It should be noted that Price/Earnings ratio (P/E) was 12-24 (Tabl. 4) and was lower than DJIA and NASDAQ (where the P/E ratio average was 21.5 with annual range 5-60). This shows a trend of increasing rates, capitalization and trading.

Typically, High-tech Companies have P/E higher than that of traditional manufacturing industry. Among the leaders on the Indian stock market are IT companies: Lanco Infra - 577, Unitech - 167, HMT 297, Cambridg Sol - 320, while some others include (Znari Inds - 1.5, Tata Steel - 7.8, Varum Ship - 5.5). (The Financial Express. 2007, Jan 25.)

Traditionally, the Asian markets are the first to respond to changes in the American financial market, but in 2006 they seemed to be less vulnerable compared to the 1990s. The share of emerging markets in the flow of global private investment capital kept growing (in 2003, it amounted to \$3.4 bln. and in 2006 it was $\$ 22$ bln.) (The Economic Times, 26 Jan 2007). It was as a result of increasing economic growth $(7-12 \%)$ and stability in the BRICS emerging markets, growing competitiveness and market capitalization (profitability of emerging markets was 2.67 times higher than for the developed ones, at $23 \%$ vs $8.6 \%$ ). Compared to 2006 , in 2007 Russian market capitalisation grew by $50 \%$ - from $\$ 1$ bln. to $\$ 1.5$ bln. Indian market capitalisation on NYSE and BSE amounted to $\$ 1.6$ trln. Stock market sectoral structure 
had changed towards processing industries and IT. Thus, in 2007, the sectoral capitalisation of the oil and gas sector on the Russian stock market decreased from $60 \%$ to $48.6 \%$, the share of the finance sector increased to $13.8 \%$, metallurgy industry to $13.7 \%$, electric-power industry to $13.4 \%$, telecommunications to $7.9 \%$.

During the period 2007-2008 the instability of interest rates on American and European markets continuously contributed to the global liquidity crisis. However, the effect of this impact was not proportional: the correlation ratio between developed markets and markets of BRICS was at 0.5 (see Table 4), in 1997-2007 it was 0.2-0.3. It reduced by $30 \%$ due to the increased stability of the BRIC markets. The international capital market responded adequately, to the strengthening of the BRIC economies and financial sectors, which became significantly more sustainable and stable with more confident global investors.

In 2007 - a year preceding crisis - qualitative fundamental changes took place on the global capital market. Companies of developing countries became the principal global investors. Their asset acquisition deals totalled $\$ 130.5$ bln. Companies from developed countries completed mergers and takeovers (M\&A) on emerging markets totalling $\$ 128.6$ bln. (Russian Business Consulting 2008: 16). A new investment focus for emerging market countries formed - the processing and High-Tech industries of developed countries. While in the 1990's the main inflow of investment was into oil and gold-mining companies, from 2007 the focus of interest shifted to assets of global metallurgy, automobile, aviation and electronics companies (Accelor, $E A D S, G M)$. At the same time there was growing competition between companies from developing economies on developed markets for High-Tech, leading edge, innovative technology, which was one of the last of the competitive advantages of developed economies.

\subsection{Changes in the BRICS' investment strategies in 2000-2007}

The BRICS investment strategies on the global capital market in 2000-2007 were quite different. While Chinese and Russian companies were still carrying out M\&A in accordance with government initiatives, India's strategy favoured the conversion of major national companies into MNCs (Tata, Wipro, and Infosys Technologies). The period featured competition between world financial centres and a developing periphery which modified the national asset protection strategy. Developed countries erected barriers and limited investments into their High-Tech, automobile and building industries, harbours and port terminals from the United Arab Emirates, South Korea and BRICS countries. The countries of the BRICS block responded by introducing limitations on the volume of foreign investment into primary industries, defence industry and natural monopolies. Under such conditions only $30 \%$ of all M\&As with companies from developed countries proved to be successful in BRICS (Russian Business Consulting 2008). Government protection measures weren't solely to blame, but also poor marketing by Western MNCs, which failed to identify the specific features of different segments of the emerging market economies.

\subsection{BRICS in the Financial and Economic Crisis of 2008-2009}

The Globalised world economy responded to the crisis in the US economy by reducing exportimport deals with the BRICS countries. The World economy reached peak crisis in the middle of 2008. In 2008 the world GDP reduced by 6\%, in developed countries it went down by 8\%, in developing and emerging economies - by $4 \%$, while the fall in the seven leading Eastern countries was half that of the "G7" (IMF World Economic Outlook Update June 17, 2011).

There are three reasons why the global crisis affected BRICS economies so deeply:

1. The economic structure of the BRICS group was based on different export markets. China focused on consumer goods, India - on IT technology and its components, Brazil - on agriculture, Russia on hydrocarbon raw materials, and the RSA specialised on various natural resources. Their economies were export dependant, state budgets and total reserves including gold depended on the capacity and state of the economies in export markets. Since the USA regained (2016) their leading position in the World economy lost in 2013, the American economy became one of the main World traders and a price target for global markets of real and financial markets. As well as a key consumer for PRC, India, Brazil and RSA, US commodity markets directly affected their 
trade balances, causing deficits. Public and private companies received diminished currency profits, resulting in decrease in national investment and volumes of production.

2. A significant share of the financial and real assets of the BRICS countries belongs to companies and institutions of the financial centre. The crisis forced them to withdraw the assets in favour of parent companies, thus resulting in the fall of share prices, stock exchange indexes and increased pressure on national currency.

3. Due to the fact that spreads always grow in a crisis, international financial markets became less available for borrowers and issuers in BRICS countries.

All the BRICS countries as well as the other G20 countries initiated their own anti-crisis policies. However, there were some common policies. Common policies included state support to financial and manufacturing sectors, promotion of internal demand and measures to prevent national currency strengthening. Specific policies in Russia included a focus on leading social programmes, surplus money sterilization, prevention of inflation growth, in China - national programmes to develop high technology, in India - focus on tax and monetary policy, in Brazil - tax cuts and national investment into infrastructure. The global financial economic crisis tested the stability of the BRICS financial and economic systems. Not only did these systems manage to overcome the crisis due to their hefty foreign exchange reserves including gold and the efficiency of their anti-crisis programmes, but it also proved that Chinese and Russian monetary policies adopted in 2000 aimed at accumulation of significant foreign currency reserves, binding surplus profits from export, were successful.

\subsection{The BRICS market during the post-crisis period}

The global financial and economic crisis of 2008-2009 hit all segments of the world financial markets. The average market reduction in volume and profitability amounted to $30 \%$. However, emerging markets become even more stable as proved by the correlation coefficient (see Table 6).

Table 6. Major stock market indexes and financial indicators, Nov. 29, 2012

\begin{tabular}{|c|c|c|c|c|}
\hline \multicolumn{2}{|r|}{ Indexes } & Changes between high/low, & Dividend Yield, & $\mathrm{P} / \mathrm{E}$ \\
\hline \multirow{3}{*}{$\begin{array}{l}\text { Dow Jones Stock } \\
\text { Indexes }\end{array}$} & Global Dow* & 5,8 & 5,38 & 12 \\
\hline & BRIC 50* & -1.6 & 2.93 & 16 \\
\hline & $\mathrm{DJ}^{* * *}$ & $\mathrm{~N} / \mathrm{A}$ & 2.7 & 13.6 \\
\hline \multicolumn{2}{|c|}{ All counties world ${ }^{* *}$} & 6.1 & 2.90 & 14 \\
\hline \multicolumn{2}{|c|}{ World Developed markets** } & 8.1 & 2.8 & 14 \\
\hline \multicolumn{2}{|c|}{ Stoxx Europe $600^{*}$} & 15.1 & 13.0 & \\
\hline \multicolumn{2}{|c|}{ Emerging markets ${ }^{* *}$} & -0.4 & 2.9 & 12 \\
\hline \multicolumn{2}{|c|}{ EMLatin America** } & -9.1 & 3.50 & 15 \\
\hline \multirow[t]{3}{*}{ USA } & USA $^{* * *}$ & $\mathrm{~N} / \mathrm{A}$ & 2.2 & 15.7 \\
\hline & US Broad market ${ }^{* *}$ & 12.3 & 2.10 & 15 \\
\hline & S\&P500*** & $\mathrm{N} / \mathrm{A}$ & 2.6 & 15.0 \\
\hline \multirow[t]{3}{*}{ UK } & $\mathrm{UK}^{* * *}$ & 6.9 & 8.7 & \\
\hline & $\mathrm{UK}^{* *}$ & 4.6 & 3.80 & 11 \\
\hline & FTSE 100* & $\mathrm{N} / \mathrm{A}$ & 3.5 & 12.4 \\
\hline \multirow[t]{2}{*}{ Germany } & Germany*** & $\mathrm{N} / \mathrm{A}$ & 3.2 & 11.8 \\
\hline & DAX30*** & $\mathrm{N} / \mathrm{A}$ & 3.5 & 13.4 \\
\hline \multirow[t]{3}{*}{ Japan } & Japan ** & 0.8 & 2.60 & 21 \\
\hline & Japan $^{* * *}$ & $\mathrm{~N} / \mathrm{A}$ & 2.4 & 14.3 \\
\hline & Nikkei Stock Average* & 9.3 & 11.2 & \\
\hline S. Korea & Hang Seng*** & $\mathrm{N} / \mathrm{A}$ & 3.3 & 11.1 \\
\hline \multirow[t]{3}{*}{ China } & China $^{* * *}$ & $\mathrm{~N} / \mathrm{A}$ & 3.9 & 7.6 \\
\hline & 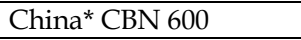 & -19.0 & -10.3 & \\
\hline & China $^{* *}$ & 4.3 & 3.10 & 10 \\
\hline \multirow[t]{3}{*}{ Brazil } & Brazil $^{* * *}$ & $\mathrm{~N} / \mathrm{A}$ & 3.8 & 13.7 \\
\hline & Bovespa, Brazil* & 1.7 & 1.9 & \\
\hline & India $^{* *}$ & 6.7 & 1.4 & 15 \\
\hline \multirow[t]{2}{*}{ India } & Sensex, India* & 16.3 & 24.0 & \\
\hline & India*** & $\mathrm{N} / \mathrm{A}$ & 1.5 & 17.3 \\
\hline \multirow[t]{3}{*}{ Russia } & Russia** & -8.0 & 3.70 & 5 \\
\hline & Russia*** & $\mathrm{N} / \mathrm{A}$ & 4.3 & 5.8 \\
\hline & RTS, Russia* & -8.2 & 2.8 & \\
\hline
\end{tabular}

www.jbrmr.com A Journal of the Academy of Business and Retail Management (ABRM) 


\begin{tabular}{|c|l|c|c|c|}
\hline \multirow{2}{*}{ RSA } & RSA $^{* *}$ & 13.8 & 3.20 & 15 \\
\cline { 2 - 5 } & RSA $^{* * *}$ & N/A & 3.6 & 15.5 \\
\hline
\end{tabular}

* (a. The Wall Street Journal. Nov.30-Dec.2, 2012.)

** (b. The Wall Street Journal. Nov.30-Dec.2, 2012.)

*** (Financial Times. Dec.1-Dec.2, 2012. - P.25. Thomson Reuters)

Let us briefly consider the trends of different global capital market segments during the relatively stable month of November 2012. The last week of the month was characterized by the simultaneous growth of key joint stock indexes S\&P $500(+0.51 \%)$, FTSE $100(+0.82 \%)$, FTSE Eurofirst $300(+0.80 \%)$, Nikkei 225 Average (+0.85\%) (Global Financial Stability Report 2011: 21). American and British indexes were rising, due to good results in the growth of bills of exchange and a fall in unemployment. However, this rising trend was moderated by the need to reduce budget expenditures and tax revenues in the USA, while in Britain it was mostly a result of unresolved systematic EU tasks, with the latter destabilizing the German CAC 40 Index $(+0.33 \%)$. The Japanese index was growing simultaneously with the American and British, yielding as much as the other growing Asian indexes (+0.85\% vs $1.4 \%)$.

The years 2010-2012 were characterized by a sluggish recession and the anticipation of the second crisis wave. The EU could neither overcome the debt crisis of the Mediterranean countries, nor catch up with technological developments. With all its flexibility, High-tech advances and diversity, the US economy was suffering from two deficits - balance of payments and national debt.

Under such conditions world product and financial markets were rather vulnerable. The World economy had no alternative sources of growth. The middle class in the developed countries could not or would not support consumption levels sufficient to provide for employment in leading industries. Emerging markets had also exhausted their potential as a source of world economic growth.

\section{Discussion of the present and future of the BRICS by its participants}

\subsection{The BRICS: a view from Brazil}

Brazilian researchers consider the present and future of the BRICS from two perspectives: strategies for the future development and tactical benefits from common markets. Some authors view the BRICS as one of the alternatives brought about by the changes in the structure of global politics and economy. They focus on the comparative analysis of the developing systems of the five countries and trends in science, technology and innovation. This group of experts emphasizes the benefits of the economic transition "from copyrighting to creating" (Cassiolato, J. Eduardo, 2009).

However, having analyzed the developing systems of these five countries, some authors doubt the ability of the BRICS to dominate long term. The reason for such doubts is the inability of their economies and technologies to innovate (Jones, S., 2015; Jose Eduardo Cassiolato, 2009). Other Brazilian analysts see the BRICS as a force, which is capable of building an alternative stable international economic and political system (Maria Regina Soares de Lima, 2012; Francesca Beausang, 2012). Researchers explain the consequences of the global crisis and evaluate the efficiency of the anti-recessionary measures and also consider the potential obstacles to their further economic growth (João Paulo dos Reis Veloso, 2009). In his book Paulo Borba Casella (Paulo Borba Casella, 2011) studies the problems connected with the latest controversial experiences of the countries, innovative trends, in India, the Republic of South Africa and China, institutional models of management in the BRICS and its international legal regulation.

\subsection{The BRICS: a view from China}

Chinese authors focus on the strengths of the BRICS and the economic and structural benefits of its model. It was a particularly popular research topic in 2011-2012 and was widely discussed in the works on contemporary international relations, foreign trade, relations between China and South African Republic (By Guo Yan, 2012; He Wenping, 2011).

The BRICS offered new mechanisms and structures for cooperation between the member countries (BRICS Cooperation Mechanism) (Wu Hongying, 2011), which now serve as a non-government communication platform for the BRICS Summits and Ministerial meetings. Besides, the BRICS have clearly defined spheres of cooperation. It is expected that the BRICS will carry out financial reforms in order to comply with the requirements of the international financial system and international financial institutions (IMF, World Bank) and increase openness and transparency (Wu Hongying, 2011. P.77). Combined efforts and cooperation between the BRICS countries, the IMF and the World Bank and 
strengthening relationships between the BRICS and the West would help transitional economies and convert regional powers into global ones. The first practical step, which has been realized, was a currency swap agreement between 4 leading banks.

A lot of the research was devoted to the role of cooperation and the BRICS model in achieving economic stability (Lin Yuegin, 2010, vol.1). Politicians and experts on the BRICS think that the future of the alliance depends on the resolution of developmental problems. The article by Yuegin Lin (Lin Yuegin, 2010) lays down a theoretical basis and offers various trajectories for this development.

\subsection{The BRICS: a view from India}

Indian economists consider the BRICS through the prism of "green economy" (Yoginder K. 2011, 2012) and study different models of cooperation and integration of the Indian economy into the global economy. One of the popular points of view is that China and India have to divide economic spheres in order to build a successful economic model. India, as the country with plentiful manpower, can focus on manufacturing, while China should concentrate more on the delivery of services to the domestic markets. Indian researchers have been cautious in their assessment of the influence that India has on the world economy (Jayati Ghosh, 2016). In general, it is considered that the Indian economy (Ruchir Sharma, 2013. $a, b)$ is more vulnerable than other BRICS countries to the outflow of foreign capital. Unlike other BRICS countries, India has not benefited from the depreciation of national currency, as it is not a main exporting country.

\subsection{The BRICS: view from South Africa}

After the accession of the Republic of South Africa to the BRICS in 2011, its experts developed their own vision of the future of the republic in the BRICS and in the African region. They state that Africa needs integration and that the Republic of South Africa can lead the integration process with the help of the alliance. Secondly, the reform of global governance should extend to international trade, development, energy and climate change. "Just as global decision-making no longer simply flows outward from the West, trade and investment patterns are also broadening" (Miller M Matola, 2012). The majority of authors also emphasize the importance of restructuring the global economic system: "developing economies should be adequately represented in G20, IMF and World Bank positions".

\section{Conclusions}

The post-crisis development of the BRICS is characterized by the growth in financial risk. Economic growth rates in China as a driving force for the world economy will inevitably reduce due to the growth of inflation, wages and a decrease in labour productivity. High net present value (NPV) of 15$20 \%$ will be more and more difficult to ensure by the high level of internal rate of return (IRR) from investment projects. Growth potential will be restrained by the growth of total reserves including gold due to the low growth rates in the financial centre and government expenditures on modernisation. This trend for interest rate decline will be replaced by their increase, since maintaining low rates in the financial centre for a long time stimulates demand for the more risky assets of emerging markets. There is no insurance against asset stripping by TNCs and share funds as a result of fear of default in Greece, Portugal, Ireland, Italy and the USA sweeping the world banking system.

In 2000 a rapid inflow of capital into the BRICS debt instruments from international share funds led to a quality decrease in expert evaluation and investment ratings. Issuances of corporate bonds have reached a record level (since 2009) in the $1^{\text {st }}$ quarter of 2011 of $\$ 65$ bln. (IMF WEO, 2011) and may replace high-yielding US corporate bonds. These financial instruments already have similar market capitalization, lower leverage and higher profit ratio. A new wave of investment into BRICS company capital will destabilize money markets and distort the rates. The fast growing Chinese real estate market also has a destabilizing influence on the global investor. It was this sector of economy that contributed to the financial crisis in Japan in 1990s.

The BRICS financial sector still has not dealt with deep and fundamental problems of stability and reliability of their banking systems. Solving these problems will strengthen the BRICS financial position and ambitions in global economy and politics. 


\section{Research limitation and direction for further research}

Global money, debt and capital markets consist of developed and developing, regional and national segments. In the global financial and economic environment they are interconnected by money flows, benchmarks and correlation. Determinism of the behaviour of its participants has geo-economic, macroeconomic, microeconomic, marketing and geopolitical nature. This research aims at revealing the regularities of the development of BRICS markets. However, it is extremely difficult to consider all factors which affect market development as it requires complex fundamental technical analysis. Economic and mathematical modelling of extensive statistical sample might level subjective judgment, but the details of deals and transactions are confidential.

Further research in this area should be directed at developing an integrated approach to the analysis and identification of trends, presenting recommendations to the monetary authorities on the regularities of markets, developing proactive measures to withstand shocks of the markets of real and financial assets.

\section{References}

Beausang, F. Globalization and the BRICs: Why the BRICs Will Not Rule the World For Long. - N.Y.: Palgrave Macmillan, 2012. - 219 p.

BIS Quarterly Report 2006, June A82 <http://www.bis.org/publ/qtrpdf/r_qt0612.htm> (accessed 12 November 2011)

BIS Quarterly Review, 2011. June. www.bis.org /publ/qtrpdf/r_ga1106.pdf\#page=110 (accessed 06 September 2011)

BIS Quarterly Review, 2016, pp. A86-A90; A113-A117.

BIS Quarterly Review, September 2016 A5 http://www.bis.org/publ/qtrpdf/r_qt1512e.htm (accessed 09 October 2017)

BIS 80th Annual Report, Basel, 2010. - P. 47. http://www.bis.org/publ/arpdf/ar2010e4.pdf (accessed 09 October 2017)

By Guo Yang. BRICS - a new model. - China Academic Journal of electronic publication House, 2012. P.32-33. New model of cooperation mechanism. http://cnki.net

Bloomberg/Top 40: IND. http://www.bloomberg.com/quote/TOP40: IND (accessed 09 November 2012)

Bloomberg/quot/indexes: IND. www.bloomberg.com/quot/indexes/: IND. (accessed 17 March 2015)

Bloomberg professional, (accessed 26 June 2015)

Bloomberg professional, (accessed April 29, 2015)

Cassiolato, J. Eduardo. BRICS and Development Alternatives: Innovation Systems and Policies. Chichester, UK: 2009, Virginia Vitorino Anthem Press. - 198 p.

http:/ / books.google.ru/books?id=UtiQ7DnV_lcC\&printsec=frontcover\&hl=ru\&source=gbs_ge_summar $\mathrm{y} \_\mathrm{r} \& \mathrm{cad}=0 \# \mathrm{v}=$ onepage $\& \mathrm{q} \& \mathrm{f}=$ false $]$

Factsheet: FTSE/JSE TOP 40 Index, 2015/. - P.2.

Financial Times, Jan.20, 2007. - P.10-11.

Financial Times. - 2007, Jan 20. - P.12.

Green Revolution. The New Oxford Companion to Economics in India, Vol.1, 301 p., Oxford University Press, 2012.

He Wenping. The Making of BRICS: South Africa Invigorates the New Cooperation Mechanism. - China and World. No1, 2011. - PP.7-9. China Academic Journal of electronic publication House. www.chinafrica.cn. http://cnki.net).

International Financing Review 2006. Sept. 9. - PP.91-92, 99-100.

$<$ http:/ / themecraft.net/www/ifree.com> (accessed 09 November 2014)

International Financing Review, 2006, Sept. 16. - P.111.

International Financing Review 2006. Sept., 23 - P. 88; P. 104, 106. http://themecraft.net/www/ifree.com (accessed 12 November 2011)

International Financial Statistics 2002. PP.186-193, 240-243, 450-453, 740-745, 914-921.

International Financial Statistics 2006. PP. 488-493, 264-267, 808-813, 198-207, 1006-1019, 138-143.

International Banking and financial market developments, Basle, May 1997. - P.10.

http:/ / www.bis.org/publ/r_qt9705.pdf (accessed 09 October 2017) 
IMF WEO Update June 17, 2011 <http://www.imf.org/external/pubs/ft/weo/2011/update/02/> (accessed 09 December 2014)

IMF World Economic Outlook, P. 2. Update June 17, 2011. www.imf.org (accessed 10 September 2011)

Jayati Ghosh. India's Tiny Impact on the World Economy. - The New York Times. - June 21, 2016.

http:/ / www.nytimes.com/roomfordebate/2012/05/11/have-the-bric-nations-lost-their-

momentum/indias-impact-on-the-world-economy-is-still-tiny

http://www.jnu.ac.in/FacultyStaff/ShowProfile.asp?SendUserName=jayati).

Jayati Ghosh. India's Tiny Impact on the World Economy. - The New York Times. - June 21, 2016.

http://www.nytimes.com/roomfordebate/2012/05/11/have-the-bric-nations-lost-their-

momentum/indias-impact-on-the-world-economy-is-still-tiny

http://www.jnu.ac.in/FacultyStaff/ShowProfile.asp?SendUserName=jayati).

Jim O`Niel, Dominic Wison, Roopa Purishothaman, Anna Stupitska. Goldman Sachs Economic Research. - Global Economic Paper No 134, 1'st December 2005. - 24 p. https:/ / portal.gs.com)

João Paulo dos Reis Veloso. A Crise Global e o novo papelmundial dos Brics (The Global Crisis and the New Role of the BRICs). - Rio de Janeiro: J. Olympio, 2009. http://pt.wikipedia.org/wiki/João_Paulo_dos_Reis_Veloso

Jones, S. BRICs and Beyond: Lessons on Emerging Markets. 2015. http:/ / books.google.ru/books?id=uy8iaN7J9vYC\&printsec $=$ frontcover\#v=onepage\& $q \& f=$ false

Jose Eduardo Cassiolato, Virginia Vitorino. BRICS and Development Alternatives: Innovation Systems and Policies. - Anthem Press, 2009. - 198 p.

http:/ / books.google.ru/books?id=UtiQ7DnV_lcC\&printsec=frontcover\&hl=ru\&source=gbs_ge_su mmary_r\&cad $=0 \# \mathrm{v}=$ onepage\& $\mathrm{q} \& \mathrm{f}=$ false

KAM, 2011 (KEI and KI Indexes). - <http://info.worldbank.org/etools/kam2/KAM_page5.asp> (accessed 13 September 2011)

Maria Regina Soares de Lima. OsBrics e aOrdem Global (The BRICS and the Global Order). São Paulo: Editora FGV]. In book: Francesca Beausang. Globalization and the BRICs: Why the BRICs Will Not Rule the World For Long. - Palgrave Macmillan, 2012.

Miller M. Matola. The Power of BRICS Alliance. - Beijing Review, May 2012, pp.10-11.

Paulo Borba Casella. Brics - Brasil, Rússia, Índia, China e África do Sul - Uma perspectiva de cooperaçãointernacional (BRICS - Brazil, Russia, India, China and South Africa - A Perspective of International Co-operation). São Paulo, 2011: Editora Atlas.

http://www.direito.usp.br/english/docentes/internacional/din_docentes_pbc_01.php

Russian Business Consulting 2008, No.16. - http://www.rbcinfosystems.ru/ir/2008.pdflast (accessed 05 October 2013)

RSA. http://www.jse.co.za/Home/Market_Data.aspx 12.11.2012.

Sharma, Ruchir, "Broken BRICs". Foreign Affairs. Council on Foreign Relations. Retrieved 14 May 2013.

Sharma, Ruchir "Hitting the BRIC Wall". Time Magazine. Retrieved 14 May 2013.

The World Bank Data <http:/ / data.worldbank.org/> (accessed 12 September 2011).

SIX financial information. WSJ Market Data Group. S\&P Dow Jones Indexes. - Nov.30-Dec.2, 2012. - P.27.

The Wall Street Journal, MSCI Indexes Barra: Morgan Stanley Index. - Nov.30-Dec.2, 2012.

The Economic Times, 26 Jan 2007. - P.2.

The Financial Express. 2007, Jan 25. - P. 24.

The Financial Times. Dec.1-Dec.2, 2012. - P.25.

Wu Hongying. On the BRICs Cooperation Mechanism. CIR Jan/Feb. 2011, p. 73-85.

Yuegin Lin. The Rise of Emerging Powers and the BRICs' Chase to Catch Up // China Economist, 2010, vol. 5, No.2. - P. 2-14

Yuegin Lin. Can Emerging Economies Catch-up Sustainably? An Analysis of BRICS`Economic Growth Pattern // China Economist, 2010, Vol. 1, No. 7, P. 106-105.

Yoginder, K. Agriculture in A Rural Urban Continuum, Indian Journal of Agricultural Economics, 2011, pp.165-177.

Yoginder, K. Green Revolution. The New Oxford Companion to Economics in India, Vol.1, Oxford University Press, 2012. - 301 p. 